\title{
AN EXACT SOLUTION OF EINSTEIN EQUATIONS FOR INTERIOR FIELD OF AN ANISOTROPIC FLUID SPHERE
}

\author{
Lakshmi S. Desai \\ Department of Mathematics, Faculty of Technology, Dharmsinh Desai University, Nadiad, Gujarat, India
}

\begin{abstract}
In this paper, an anisotropic relativistic fluid sphere with variable density, which decreases along the radius and is maximum at the centre, is discussed. Spherically symmetric static space-time with spheroidal physical 3-space is considered. The source is an anisotropic fluid.

The solution is an anisotropic generalization of the solution discussed by Vaidya and Tikekar [1]. The physical three space constant time has spheroidal solution. The line element of the solution can be expressed in the form Patel and Desai [2]. The material density is always positive. The solution efficiently matches with Schwarzschild exterior solution across the boundary. It is shown that the model is physically reasonable by studying the numerical estimates of various parameters. The density vs radial pressure relation in the interior is discussed numerically. An anisotropy effect on the redshift is also studied numerically.
\end{abstract}

Key Words: Cosmology, Anisotropic fluid sphere, Radial pressure, Radial density, Relativistic model.

\section{INTRODUCTION}

The hypothesis of local isotropy is universal in the astrophysical studies of massive bodies. This is a reasonable first approximation for matter whose dominant properties depend on chemical forces (coulomb interaction, etc.). For the majority of systems that depend fully on general relativity it is the strong interactions which take over local physics. It is well known that these strong interactions are very complex and they are highly dependent on velocity, spin and parity. Therefore it is intricate to conclude from a prior consideration alone whether strongly interacting matter is locally isotropic or not. The theoretical study on realistic stellar models suggests that stellar matter could be anisotropic at least in certain density ranges $\left[\rho<10^{15} \mathrm{~g} \cdot \mathrm{cm}^{-3}\right]$.

Additional possible source of local anisotropy would be a solid core. The calculations show that a solid state may occur for cold matter in the density range $4 \times 10^{14}-3 \times 10^{15}$ g. $\mathrm{cm}^{-3}$. However, the exact nature of such a state is not clear. Bowers and Liang [3] have made a detailed study of incompressible anisotropic fluid spheres for general relativity. According to their study, if anisotropy is present in the expected density range for relativistic stars (i.e. $\rho<$ $10^{15} \mathrm{~g} . \mathrm{cm}^{-2}$ ), effects significantly on maximum equilibrium mass and surface red-shift parameters. The knowledge of possible anisotropic properties of dense matter may prove useful in understanding the physics of neutron stars and black holes, furthermore the possible connection between quasars and relativistic compact objects.

It is implicit that the radial pressure is not equal to the tangential pressure in massive stellar bodies. Many researchers [4-8] have devoted significant attention to the problem of finding interior solutions of Einstein equations corresponding to static anisotropic fluid spheres. Patel and
Mehta [9] have obtained an exact solution for a static anisotropic fluid sphere whose physical space constant time is pseudo spheroidal. This solution is free from singularity and satisfies the reasonable physical conditions. Mak et. al [10] studied spherically symmetric and static anisotropic stellar type configurations.

Maurya et. al. [11] described anisotropic super - dense star models using charged perfect fluid distributions. A static spherically symmetric anisotropic quark matter distribution is described by Mak and Harko [12]. Thirukkanesh and Maharaj [13] have obtained general form linear equation for anisotropic matter distributions in the presence of the electromagnetic field.

In many of the above mentioned solutions the density is assumed to be uniform. However, indeed, most of the stellar objects have variable density. Hence, solutions for anisotropic fluid spheres with variable density are physically more relevant. Most of the above solutions are such that the corresponding solutions for isotropic spheres cannot be recovered from them. This situation is not desirable.

In this paper, a new exact solution for anisotropic fluid sphere with variable density distribution is discussed. In the absence of anisotropy, the solution reduces to the solution for the isotropic fluid discussed by Vaidya and Tikekar [1] for a relativistic model of a superdense star.

In most of the above cited solutions, the physical 3-space constant time is spherical. The aim of the present investigation is to find an interior solution for anisotropic fluid sphere whose associated physical 3-space constant time is spheroidal. Vaidya et. al. [1] explained the spacetimes, with physical 3-space spheroidal, in great detail. They have shown that the geometry of such a space-time is described by the metric Patel and Desai [2]. 
$d s^{2}=e^{\gamma} d t^{2}-\left[1-\frac{k r^{2}}{R^{2}}\right]\left[1-\frac{r^{2}}{R^{2}}\right]^{(-1)} d r^{2}$ $-r^{2}\left(d \theta^{2}+\sin ^{2} \theta d \emptyset^{2}\right)$

where, $\gamma$ is an arbitrary function of $\mathrm{r}$. Here $\mathrm{k}$ and $\mathrm{R}$ are constants, $\mathrm{k}<1$. The line-element 1.1 is regular at all points where $r^{2}<R^{2}$. The coordinates are denoted as $x^{1}=r, \quad x^{2}=$ $\theta, \mathrm{x}^{3}=\phi, \mathrm{x}^{4}=\mathrm{t}$. When $\mathrm{k}=0$, the physical 3-space constant time becomes spherical.

\section{THE FIELD EQUATIONS}

Metric 1.1 forms Einstein field equations for a static anisotropic fluid sphere of the space-time metric. Einstein field equations in the presence of matter are

$R_{i k}-\frac{1}{2} g_{i k} R=-8 \pi T_{i k}$

The energy momentum tensor is considered here as

$T_{i k}=\rho v_{i} v_{k}+p h_{i k}+t_{i k}$

where, $v_{i}=e^{\gamma / 2} \delta_{i}^{t}$ denotes the four velocity, $\rho$ is the energy density, $\mathrm{p}$ is the isotropic pressure, $h_{i k}=v_{i} v_{k}-(1 / 2) g_{i k}$ is the projection tensor and $t_{i k}$ is the anisotropic pressure (stress) tensor given by

$t_{i k}=\sqrt{3} S(r)\left[c_{i} c_{k}-(1 / 3) h_{i k}\right]$

where, $|S(r)|$ is the magnitude of the stress tensor and $c_{i}=e^{\lambda / 2} \delta_{i}^{r}$ is a unit radial vector. The non-vanishing components of $T_{k}^{i}$ given by 2.2 can be mentioned as

$T_{4}^{4}=\rho, T_{1}^{1}=-\left[p+\frac{2 S}{\sqrt{3}}\right]$,

$T_{2}^{2}=T_{3}^{3}=-\left[p-\frac{s}{\sqrt{3}}\right]$

The tangential pressure $p_{\perp}$ and the radial pressure $p_{\mathrm{r}}$ are expressed as,

$p_{r}=p+\frac{2 s}{\sqrt{3}}, \quad p_{\perp}=p-\frac{s}{\sqrt{3}}$

Thus, $S=\frac{1}{\sqrt{3}}\left[p_{r}-p_{\perp}\right]$

With the help of the equations 1.1 to 2.5 the Einstein field equations become

$$
\begin{aligned}
8 \pi \rho= & \frac{3(1-k)}{R^{2}}\left[1-\frac{r^{2}}{R^{2}}\right]\left[1-\frac{k r^{2}}{R^{2}}\right]^{(-2)} \\
8 \pi p_{r}= & {\left[\frac{\gamma^{\prime}}{r}\left(1-\frac{r^{2}}{R^{2}}\right)-\frac{(1-k)}{R^{2}}\right]\left(1-\frac{k r^{2}}{R^{2}}\right)^{-1} } \\
8 \pi p_{\perp}= & {\left[\frac{\gamma^{\prime \prime}}{2}+\frac{\gamma^{\prime 2}}{4}+\frac{\gamma^{\prime}}{2 r}\right]\left(1-\frac{r^{2}}{R^{2}}\right)\left(1-\frac{k r^{2}}{R^{2}}\right)^{-1}-} \\
& \frac{r(k-1)}{R^{2}}\left[\frac{\gamma^{\prime}}{2}+\frac{1}{r}\right]\left(1-\frac{k r^{2}}{R^{2}}\right)^{-2}
\end{aligned}
$$

In this paper, an overhead dash denotes differentiation with respect to $\mathrm{r}$.

\section{A SOLUTION OF THE FIELD EQUATIONS}

Here there is a system of three equations $2.6-2.8$ for four unknown functions $\mathrm{p}_{\mathrm{r}}, \quad p_{\perp}, \rho$ and $e^{\gamma}$. So there is one free parameter. In order to get an explicit solution, one more restriction is added on the behavior of these functions is set. In this paper the anisotropy $p_{r}-p_{\perp}$ is specified.

From the equations 2.7 and 2.8 it is necessary to see

$$
\begin{aligned}
& 8 \pi \sqrt{3} S\left(1-\frac{k r^{2}}{R^{2}}\right)\left(1-\frac{r^{2}}{R^{2}}\right)^{-1}=\frac{k(k-1)\left(r^{2} / R^{2}\right)}{R^{2}\left(1-\frac{r^{2}}{R^{2}}\right)\left(1-\frac{k r^{2}}{R^{2}}\right)}+ \\
& \frac{\gamma^{\prime}}{2}\left[\frac{1}{r}+\frac{r(1-k)}{R^{2}\left(1-\frac{r^{2}}{R^{2}}\right)\left(1-\frac{k r^{2}}{R^{2}}\right)}\right]-\frac{1}{2}\left[\gamma^{\prime \prime}+\frac{{\gamma^{\prime}}^{\prime 2}}{2}\right]
\end{aligned}
$$

The equations 2.6 illustrates that the fluid density is calculated by the curvature of the physical 3-space. Hence, conventional state equation is replaced by the geometrical requirement for a spheroidal physical three space.

The anisotropy function $\mathrm{S}$, the linear equation 3.1 can be solved if a suitable form is assumed. The relation

$8 \pi \sqrt{3} S=\frac{-\beta r^{2}}{R^{4}\left(1-\frac{k r^{2}}{R^{2}}\right)^{2}}$

where $\beta$ is an arbitrary constant is assumed.

$F=e^{\gamma / 2}$
$u^{2}=\frac{k}{(k-1)}\left(1-\frac{r^{2}}{R^{2}}\right) \quad$ if $k<0 \quad$ or
$u^{2}=\frac{k}{(1-k)}\left(1-\frac{r^{2}}{R^{2}}\right) \quad$ if $0<k<1$

The equation 3.1 now reduces to the convenient form

$\left(1-u^{2}\right)\left[d^{2} F / d u^{2}\right]+u[d F / d u]+\left[1-k+\left(\frac{\beta}{k}\right)\right] F=0$

The points $u= \pm 1$ are regular singular points whereas rest are regular points of this differential equation. If a series solution is looked for, of this equation in the form $F=$ $\Sigma A_{i} u^{i}$, the following recurrence relation for the coefficients $A_{i}$ is obtained:

$(n+1)(n+2) A_{n+2}=\left[n^{2}-2 n+k-\left(\frac{\beta}{k}\right)\right] A_{n}$

If the parameters $\mathrm{k}$ and $\beta$ have values such that

$n^{2}-2 n+k-1-\left(\frac{\beta}{k}\right)=0$

states $\mathrm{n}$ integral solutions from any of these two sets

$\left(A_{0}, A_{2}, A_{4}, A_{6}, \ldots \ldots\right),\left(A_{1}, A_{3}, A_{5}, A_{7}, \ldots \ldots\right)$

These two sets contain finite elements and a finite polynomial is obtained in the solution series using the corresponding terms. The simplest relation between $\mathrm{k}$ and $\beta$ is $k=-1-\sqrt{1+\beta}$ which corresponds to $\mathrm{n}=3$. It is 
assumed that $1+\beta$ is positive. The solution of 3.3 corresponding to this value of $\mathrm{k}$ can be expressed as

$F=A z\left(1-\frac{2 k z^{2}}{3(k-1)}\right)+B\left(1-\frac{k z^{2}}{k-1}\right)^{3 / 2}$

here, $\mathrm{z}^{2}=1-\left(\mathrm{r}^{2} / \mathrm{R}^{2}\right)$ and $\mathrm{A}$ and $\mathrm{B}$ are arbitrary constants of integration. Here it should be noted that other closed form solutions of the equation 3.3 can also be obtained.

The physical parameters $\rho, p_{r}$, and $p_{\perp}$ for the solution 3.4 are determined as follows:

$8 \pi \rho=\frac{3(1-k)\left(1-\frac{k r^{2}}{3 R^{2}}\right)}{R^{2}\left(1-\frac{k r^{2}}{R^{2}}\right)^{2}}$

$8 \pi p_{r} R^{2}\left(1-k+k z^{2}\right)\left[A z\left\{1-\frac{2 k z^{2}}{3(k-1)}\right\}+B\{1-\right.$

$\left.\left.\frac{k z^{2}}{k-1}\right\}^{3 / 2}\right]=A z\left[k-3+\frac{2 k(7-k) z^{2}}{3(k-1)}\right]+B\{1-$

$\left.\frac{k z^{2}}{k-1}\right\}^{1 / 2}\left(k-1+\frac{k(7-k) z^{2}}{(k-1)}\right)$

$8 \pi p_{\perp}=8 \pi p_{r}+\frac{\beta\left(1-z^{2}\right)}{R^{2}\left(1-k+k z^{2}\right)}$

It should be noted that at the centre $\mathrm{r}=0, p_{r}=p_{\perp}$. The density $\rho$ and the radial pressure $p_{r}$ at the centre $(\mathrm{r}=0)$ attain the values

$8 \pi \rho_{0}=\frac{3(1-k)}{R^{2}}$

$8 \pi p_{r_{0}}=f(A, B)$

where, $\mathrm{f}(\mathrm{A}, \mathrm{B})$ is defined by

$f(A, B) R^{2}\left[\frac{A(3-k)}{3(1-k)}+\left(1-\frac{k}{k-1}\right)^{3 / 2}\right]=\frac{A\left(k^{2}+2 k+9\right)}{3(k-1)}+$

$B\left(\frac{5 k+1}{k-1}\right)\left(1-\frac{k}{k-1}\right)^{1 / 2}$

From 3.8 and 3.9 it is easy to see that

$8 \pi\left(\rho_{0}-3 p_{0}\right)=g(A, B)$

where, $\mathrm{g}(\mathrm{A}, \mathrm{B})$ is defined by

$\frac{1}{6} g(A, B) R^{2}\left[\frac{A(3-k)}{3(1-k)}+B\left(1-\frac{k}{k-1}\right)^{3 / 2}\right]=\frac{A\left(k-k^{2}-5\right)}{3(k-1)}+$

$B\left(\frac{1+2 k}{1-k}\right)\left(1-\frac{k}{k-1}\right)^{1 / 2}$

At the centre, the physical requirements

$\rho_{0}>0, p_{r_{0}} \geq 0, \rho-3 p_{r_{0}} \geq 0$

should be satisfied. These requirements can be expressed as

$\mathrm{f}(\mathrm{A}, \mathrm{B}) \geq 0, \quad \mathrm{~g}(\mathrm{~A}, \mathrm{~B}) \geq 0$

If the radius of fluid sphere is a, the exterior field $(r \geq a)$ is uniquely expressed by the Schwarzschild exterior metric $d s^{2}=\left(1-\frac{2 m}{r}\right) d t^{2}-\left(1-\frac{2 m}{r}\right)^{-1} d r^{2}-r^{2}\left(d \theta^{2}+\right.$ $\left.\sin ^{2} \theta d \phi^{2}\right)$ where, $\mathrm{m}$ is the mass of the sphere.

The geometry of the interior solution $(r \leq a)$ is expressed by the metric

$d s^{2}=\left[A z\left\{1-\frac{2 k z^{2}}{3(k-1)}\right\}+B\left(1-\frac{k z^{2}}{k-1}\right)^{3 / 2}\right]^{2} d t^{2}-$

$\frac{\left(1-k+k z^{2}\right)}{z^{2}} d r^{2}-r^{2}\left(d \theta^{2}+\sin ^{2} \theta d \phi^{2}\right)$

where, $z^{2}=1-\left(r^{2} / R^{2}\right)$

Any physically significant solution must satisfy the following boundary conditions:

(i) The line elements 3.15 and 3.16 should match

(ii) The radial pressure $\mathrm{p}_{\mathrm{r}}$ must vanish at the boundary $r=a$.

The above mentioned boundary conditions determine the constants $\mathrm{m}, \mathrm{A}$ and $\mathrm{B}$ as

$\frac{2 m}{a}=\frac{a^{2}(1-k)}{R^{2}\left(1-\frac{k a^{2}}{R^{2}}\right)}$

$B\left(1-\frac{2 m}{a}\right)^{-\left(\frac{1}{2}\right)}\left[(k-1)\left\{\frac{1-\frac{k a^{2}}{R^{2}}}{1-k}\right\}^{\frac{3}{2}}\left\{-\left(k^{2}+2 k+9\right)+\right.\right.$

$\left.2 k(7-k) \frac{a^{2}}{R^{2}}\right\}+\left\{\frac{1-\frac{k a^{2}}{R^{2}}}{1-k}\right\}\left\{k-3+\frac{2 k a^{2}}{R^{2}}\right\}\{5 k+1+$

$\left.\left.k(k-7) \frac{a^{2}}{R^{2}}\right\}\right]=(1-k)\left[9+2 k+k^{2}-2 k(7-k) \frac{a^{2}}{R^{2}}\right]$

$A\left(1-\frac{a^{2}}{R^{2}}\right)^{\frac{1}{2}}\left[\frac{k-3+2 k a^{2} / R^{2}}{3(k-1)}\right]=$

$\left(1-\frac{a^{2}}{R^{2}}\right)^{\frac{1}{2}}\left(1 \frac{k a^{2}}{R^{2}}\right)^{-1 / 2}-B\left(\frac{1-\frac{k a^{2}}{R^{2}}}{1-k}\right)^{3 / 2}$

From the expression 3.5 for matter density $\rho$ we see that $\rho$ stays positive throughout the configuration because $\mathrm{k}<0$. Since $\rho^{\prime}$ is negative, the density decreases from the maximum value $\rho_{0}$ at the centre as $\mathrm{r}$ increases. From 3.17 it is obvious that the mass parameter $\mathrm{m}$ is always positive.

The requirement

$\frac{\operatorname{Lim}_{(\rightarrow 0}\left(p_{r}-p_{\perp}\right)}{r}=0$

for anisotropic fluid spheres is trivially satisfied. If $\beta=0$, $\mathrm{k}=(-2)$ and the anisotropy in the pressure vanishes. In this case the above solution reduces to the solution for isotropic fluid sphere discussed by Vaidya and Tikekar [1]. Therefore this solution is a generalization of Vaidya -Tikekar solution to an anisotropic case. 


\section{DISCUSSION}

On the boundary $r=$ a density is,

$8 \pi \rho_{0}=\frac{3(1-k)}{R^{2}}\left(1-\frac{k a^{2}}{3 R^{2}}\right)\left(1-\frac{k a^{2}}{R^{2}}\right)^{(-2)}$

The ratio $\rho_{a}$ to $\rho_{0}$ is obtained. It is given by

$\mu=\frac{\rho_{a}}{\rho_{0}}=\left(1-\frac{k a^{2}}{3 R^{2}}\right)\left(1-\frac{k a^{2}}{R^{2}}\right)^{(-2)}$

It is not difficult to see that $\mu<1$. The equation 4.2 represents $\mathrm{a}^{2} / \mathrm{R}^{2}$ in terms of $\mu$ and $\mathrm{k}$ as

$\frac{a^{2}}{R^{2}}=\frac{[6 \mu-1-\sqrt{1+24 \mu}]}{6 k \mu}$

In order to have some more information about the model, a numerical study of various parameters occurring in our solution is discussed. Using the scheme outlined above, the matter density is taken as $\rho_{a}=2 \times 10^{14} \mathrm{~g} . \mathrm{cm}^{-3}$ on the boundary $\mathrm{r}=\mathrm{a}$ of the sphere. Different values of the ratio $\mu=\frac{\rho_{a}}{\rho_{0}}$ are chosen and $\rho_{0}$ is computed for each the assumed value of $\rho_{\mathrm{a}}$ and selected value of $\mu$. If a specific value is assigned to the constant $\beta$, then $k=-1-\sqrt{1+\beta}$ gives $\mathrm{k} . \beta=0.21$ is taken so that $\mathrm{k}$ is negative and taken as -2.1 . The equation 3.8 provides $\mathrm{R}$. An estimate of the radius of the sphere (a) is given by equation 4.3 . The equations 3.17 , 3.18 and 3.19 give the values of $\mathrm{m}, \mathrm{B}$ and A respectively. Equation 3.17 gives the mass $(\mathrm{m})$ in $\mathrm{kgs}$. The mass $\mathrm{M}$ of the body in $\mathrm{gm}$ is obtained from $\mathrm{M}=\mathrm{mc}^{2} / \mathrm{G}$. The values $\mathrm{f}(\mathrm{A}, \mathrm{B})$ and $\mathrm{g}(\mathrm{A}, \mathrm{B})$ can be obtained from 2.10 and 3.12. Table 2 shows the results for the various values of $\mu$.

Table 2 indicates that $\mathrm{a}, \mathrm{a} / \mathrm{R}$ and $\mathrm{m}$ are decreasing functions of $\mu$. $\mathrm{R}$ and $\mathrm{A}$ are increasing functions of $\mu$ while $\mathrm{B}$ is a decreasing function of $\mu$. The physical requirements are $f(A, B) \geq 0$ and $g(A, B) \geq 0$. From the table 2 it is clear that these requirements are satisfied for $0.55 \leq \mu<1$. For this range the maximum radius is $16.95 \mathrm{~km}$ and maximum mass is $2.6132 \mathrm{M}_{0}$. Here $\mathrm{M}_{0}$ is the solar mass given $\mathrm{M}_{0}=$ $1.475 \mathrm{~km}$. Therefore, the model permits densities of the order $2 \times 10^{14}{\mathrm{~g} . \mathrm{cm}^{-3}}^{-}$masses around three times the solar mass and radii of the order of few kilometers. Although the numerical calculations are performed for $\beta=0.21$, the method is quite general and can be used for any real value of $\beta$.

From the results 3.5 and 3.6 it is clear that an explicit relation between the density $\rho$ and the radial pressure $p_{r}$ cannot be obtained. Such a relation is discussed numerically. Let us fix $k$ and $a / R$ as $k=-2.1$ and $a / R=(0.5)$. This value of $\mathrm{a} / \mathrm{R}$ is admissible. The constant $\mathrm{A}$ and $\mathrm{B}$ can be calculated from 3.18 and 3.19. For various values of $r / R$ lying between 0 and 0.5 , the values of $\rho R^{2}$ and $p_{r} R^{2}$ are tabulated as follows:
Table -1: Density and pressure values in the interior of the sphere

\begin{tabular}{|c|c|c|}
\hline $\mathrm{r} / \mathrm{R}$ & $\rho \mathrm{R}^{2}$ & $\mathrm{p}_{\mathrm{r}} \mathrm{R}^{2}$ \\
\hline 0.04 & 9.248 & 2.160 \\
\hline 0.08 & 9.000 & 2.000 \\
\hline 0.12 & 8.850 & 1.980 \\
\hline 0.16 & 8.520 & 1.820 \\
\hline 0.20 & 8.136 & 1.640 \\
\hline 0.24 & 7.690 & 1.430 \\
\hline 0.28 & 7.230 & 1.210 \\
\hline 0.32 & 6.750 & 0.980 \\
\hline 0.6 & 6.260 & 0.751 \\
\hline
\end{tabular}

From the above table 1 it is clear that $\rho-3 p_{r}>0$ for $r / R$ lying between 0.04 and 0.36 . With the help of table 1, the graph of $\rho R^{2}$ verses $p_{r} R^{2}$ is plotted in Fig. 1. The graph shows the pressure -density relation in the interior of the sphere.

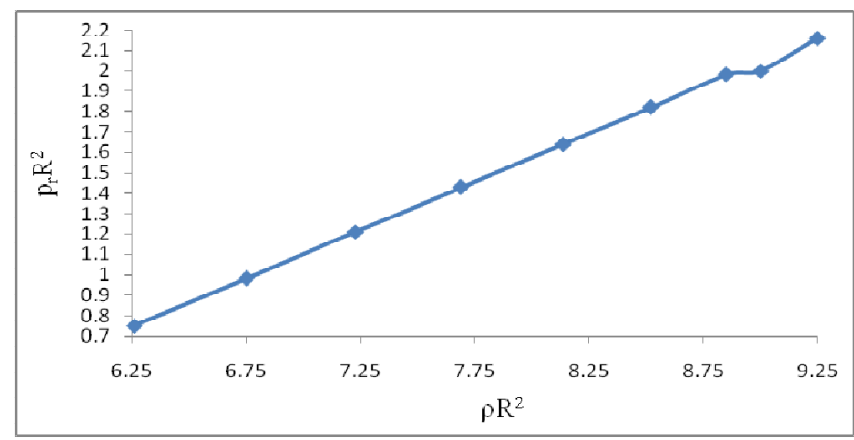

Fig -1: Graph of the pressure -density relation in the interior of the sphere

The conservation laws $T_{k ; i}^{i}=0$ for the anisotropic fluid distribution is,

$\left(\rho+p_{r}\right) \frac{\gamma^{\prime}}{2}=-p_{r}^{\prime}+\frac{2}{R}\left(p_{\perp}-p_{r}\right)$

These rules for isotropic fluid distribution provide

$\left(\rho+p_{r}\right) \frac{\gamma^{\prime}}{2}=-p_{r}^{\prime}$

By examining these equations, a supplementary term $[2 / \mathrm{r}]\left(p_{\perp}-p_{r}\right)$ represents "force" as a result of anisotropic nature of the fluid. When $p_{\perp}>p_{r}$ the force is directed outwards and for $p_{\perp}<p_{r}$ it is inwards. Compared to isotropic fluid, use of an anisotropic fluid permits construction of largely compact distributions, as the repellant force exists in the outward directed force.

Here, the physical condition $\frac{d p_{r}}{d \rho}<1$ at the centre and at the boundary is,

$\left(\frac{d p_{r}}{d \rho}\right)_{r=0}=\frac{8 \pi\left(\rho_{0}+p_{r_{0}}\right) R^{2}\left\{A\left(\frac{1+k}{1-k}\right)+\frac{3 B k}{(1-k)^{3 / 2}}\right\}}{4 k\left\{\frac{A}{3}(3-k)+\frac{B}{\sqrt{1-k}}\right\}}+\frac{\beta}{2 k(1-k)}$

and 
$\left(\frac{d p_{r}}{d \rho}\right)_{r=a}=$

$\frac{8 \pi \rho_{a}\left(1-\frac{k a^{2}}{R^{2}}\right)^{2} \frac{R^{2}}{z_{a}}\left\{A\left(1-\frac{2 k}{k-1} z_{a}^{2}\right)-\frac{3 B k}{k-1} z_{a}\left(1-\frac{k z_{a}^{2}}{k-1}\right)^{1 / 2}\right\}}{4 k(1-k)\left\{A z_{a}\left(1-\frac{2 k}{3(k-1)} z_{a}^{2}\right)+B\left(1-\frac{k}{k-1} z_{a}^{2}\right)^{3 / 2}\right\}}+$

$\frac{\beta\left(1-\frac{k a^{2}}{R^{2}}\right)}{2 k(1-k)}$

Where, $z_{a}^{2}=1-\left(a^{2} / R^{2}\right)$.

For the various values of $\mu$ lying between 0.55 and 0.9 , the values of $\left(\frac{d p_{r}}{d \rho}\right)_{r=0}$ and $\left(\frac{d p_{r}}{d \rho}\right)_{r=a}$ are mentioned in the table 2.

Table -2: Values of $\left(\frac{d p_{r}}{d \rho}\right)_{r=0}$ and $\left(\frac{d p_{r}}{d \rho}\right)_{r=a}$ for different values of $\mu$

\begin{tabular}{|c|c|c|}
\hline$\mu$ & $\left(\frac{d p_{r}}{d \rho}\right)_{r=0}$ & $\left(\frac{d p_{r}}{d \rho}\right)_{r=a}$ \\
\hline 0.9000 & 0.71696 & 0.69999 \\
\hline 0.8500 & 0.78145 & 0.76799 \\
\hline 0.8000 & 0.67505 & 0.70726 \\
\hline 0.7500 & 0.72501 & 0.76885 \\
\hline 0.7000 & 0.75870 & 0.82165 \\
\hline 0.6500 & 0.86167 & 0.93724 \\
\hline 0.6000 & 0.96304 & 1.05669 \\
\hline 0.5500 & 1.10602 & 1.21521 \\
\hline
\end{tabular}

From the table 2 it is clear that $\frac{d p_{r}}{d \rho}$ at the centre and at the boundary remains less than one for range $0.65 \leq \mu \leq 0.9$. Therefore the range of validity of the solution is $0.65 \leq \mu \leq$ 0.9 .

Now the effect of anisotropy on the surface red-shift is discussed. The red-shift is given by

$z=\left(1-\frac{2 m}{a}\right)^{(-1 / 2)}-1$

where, a is the boundary radius.

For isotropic case $\mathrm{z}$ is represented by $\mathrm{z}_{\mathrm{i}}$ and for anisotropic case $\mathrm{z}$ is represented by $\mathrm{z}_{\mathrm{a}}$. For various values of $\mu$ lying between 0.55 and $0.9, \mathrm{z}_{\mathrm{i}}$ and $\mathrm{z}_{\mathrm{a}}$ are tabulated below.

Table -3: Various values of for $\mu$ for $z_{i}$ and $z_{a}$

\begin{tabular}{|c|c|c|}
\hline$\mu$ & $\mathrm{z}_{\mathrm{i}}$ & $\mathrm{z}_{\mathrm{a}}$ \\
\hline 0.9000 & 0.04723 & 0.04877 \\
\hline 0.8500 & 0.07468 & 0.07708 \\
\hline 0.8000 & 0.10549 & 0.10880 \\
\hline 0.7500 & 0.14017 & 0.14454 \\
\hline 0.7000 & 0.17978 & 0.18520 \\
\hline 0.6500 & 0.22567 & 0.23248 \\
\hline 0.6000 & 0.27945 & 0.28772 \\
\hline 0.5500 & 0.34407 & 0.35403 \\
\hline
\end{tabular}

Table 3 shows that $z_{a}$ is always greater than $z_{i}$. Therefore, the introduction of anisotropy parameter in pressure increases in surface red-shift.

\section{CONCLUSION}

In this paper exact solution of Einstein field equations which describes the interior field of an anisotropic fluid sphere is derived. The solution satisfies reasonable physical conditions. The differential equation 3.3 can also admit exact solution for different choices of $\beta$. For example, the solution of 3.3 in which $\beta=\mathrm{k}(\mathrm{k}-1)$ is obtained. The details of this solution can be discussed on similar lines.

A system of equations $2.6-2.8$ by making a specific choice of the pressure anisotropy function $\mathrm{S}$ is solved. But this system of equations can also be solved by making a particular choice of the radial pressure $\mathrm{p}_{\mathrm{r}}$. One possible choice of $\mathrm{p}_{\mathrm{r}}$ is

$8 \pi p_{r}=f(r)\left(1-\frac{r^{2}}{a^{2}}\right)^{n}$

where $\mathrm{f}$ is an arbitrary function of $\mathrm{r}$, $\mathrm{a}$ is the boundary radius and $\mathrm{n}$ is any natural number. Here the radial pressure disappears at the boundary. So, one of the boundary conditions is already satisfied.

\section{REFERENCES}

[1]. Vaidya, P. C. and Tikekar, R., "Exact relativistic model for a superdense star" J. Astrophys. Astr., Vol. 3, No. 3, pp. $325-334,1982$.

[2]. Patel, L. K. and Desai, L. S., “A radiating black hole with internal monopole in expanding Universe", Mathematics Today, 1996.

[3]. Bowers, R. L. and Liang, E. P. T., "Anisotropic spheres In general relativity” The Astrophys. J., Vol. 188, pp. 657 $665,1974$.

[4].Consenza, M., Herrera, L., Esculpi, M., and Witten, L., "Some models of anisotropic spheres in general relativity" J. Math. Phys., Vol. 22, No. 1, pp. 118 - 125,1981.

[5]. Bayin, S. S., "Anisotropic fluid spheres in general relativity” Phys. Rev. D, Vol. 26, No. 6, pp. 1262 - 1274, 1982.

[6]. Krori, K. D., Bargohain, P., and Devi, R., "Some exact anisotropic solutions in general relativity" Cand. J. Phys., Vol. 62, No. 3, pp. 239 - 246, 1984.

[7]. Maharaj, S. D. and Maartens, R. "Anisotropic spheres with uniform energy density in general relativity" Gen. Rel. Grav., Vol. 21, No.9, pp.899 - 905,1989.

[8]. Gokhroo, M. K. and Mehra, A. L. "Anisotropic spheres with variable energy density in general relativity", Gen. Rel. Grav, Vol. 26, No. 1, pp. 75 - 84, 1994.

[9]. Patel, L. K. and Mehta, N. P., "An exact model of an anisotropic relativistic sphere" Australian Journal of Physics, Vol. 48, No.4, pp. 635 - 644, 1995.

[10]. Mak M. K., Dobson Jr., P. N., and T. Harko, "Exact models for anisotropic relativistic stars", Int. J. Mod. Phys. D, Vol. 11, No. 2, pp. 207 - 221, 2002. 
[11]. Maurya, S. K. and Gupta, Y. K., "A family of anisotropic super-dense star models using a space-time describing charged perfect fluid distributions" Physica Scripta, Vol. 86, No. 2, pp. 25009 - 25017, 2012.

[12]. Mak M. K. and Harko, T., "An exact anisotropic quark star model" Chin. J. Astron. and Astrophys., Vol. 2, No. 3, pp. $248-259,2002$.

[13]. Thirukkanesh, S. and Maharaj, S. D., "Charged anisotropic matter with a linear equation of state", Class. and Quantum Grav., Vol. 25, No. 23, pp. 235001 - 235014, 2008.

\section{BIOGRAPHY}

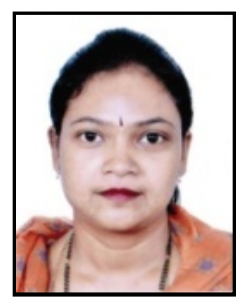

Dr. Lakshmi S. Desai obtained her doctoral degree in the field of Applied Mathematics from Gujarat University, Ahmedabad, India. She has over 17 years of extensive experience at undergraduate and postgraduate levels at Faculty of Technology, Dharmsinh Desai University, Nadiad (formerly known as Dharmsinh Desai Institute of Technology - DDIT). Her area of research is Applied Mathematics, General Relativity, Cosmology, and Visual Cryptography. 